\title{
Alternative opioids to morphine in palliative care: a review of current practice and evidence
}

\author{
M Barnett
}

This is a review of current practice of opioid use in palliative care, conducted from the perspective of a practising clinician working in the increasingly complex area of symptom control. In examining alternative opioids to morphine, choice and availability of different drugs reflect the UK perspective. Some drugs or formulations may not be available elsewhere, but the principles discussed may hopefully still be applied.

The aims of this paper are several-fold:

(1) To present an overview of available opioids.

(2) To consider factors affecting possible choice of opioid-with particular reference to the palliative care setting.

(3) To consider availability and limitations of current data which may affect evidence based decisions.

(4) To comment on areas of interest for future clinical trials.

\section{Terms of reference}

OPIOID RECEPTORS AND EFFECTS

There are three main classes of opioid receptor: mu, kappa, and delta (table 1), responsible for differing opioid effects. Opioid drugs vary in their receptor affinity, thus affecting their principal actions (table 2). The main site of action is the mu receptor, but some opioids have more complex activity.

Coventry and University of Warwick

Correspondence to: Dr M Barnett, Centre for Primary Health Care Studies, University of Warwick, Coventry CV4 7AL, UK

Submitted 8 May 2000 Accepted 31 October 2000

\section{SIDE EFFECTS OF OPIOIDS}

Side effects are common to all opioids, although to differing degrees.

- Sedation

- Hallucinations

- Constipation

Table 1 Classes of opioid receptor and response mediated

Receptor Response on activation

$\mathrm{Mu} \quad$ Analgesia, respiratory depression, miosis, euphoria, reduced gastrointestinal mobility

Kappa Analgesia, dysphoria, psychotomimetic effects, miosis, respiratory depression

Delta Analgesia

Source: Oxford Textbook of Palliative Medicine.

Table 2 Differences in opioid receptor action

- Nausea/vomiting

- Urinary retention

- Myoclonus

- Paradoxical pain

- Respiratory depression

For practical purposes, the most important side effects are sedation, nausea, and constipation.

Sedation and nausea occur particularly when starting the drug, usually temporarily, but may recur with dose increases. Nausea can be pre-empted by using a centrally acting antiemetic. This is not always necessary but advisable if the patient is already nauseated or fearful about it. Sedation is usually unavoidable but short lived (48-72 hours) among patients starting off on low doses. It may become more intractable at high dose, and there is some work on counteracting this effect with stimulants, ${ }^{1}$ although not widely practised.

Constipation, in contrast, occurs in almost every patient taking opioids and does not lessen with continued use, but can be ameliorated by aperients.

Respiratory depression, while potentially serious, is rarely clinically significant when treating pain (even among patients with respiratory impairment), as this antagonises the depressant effect. In practice respiratory depression usually occurs in opioid naïve patients after acute administration (for example, bolus intravenous dose). Tolerance develops rapidly with repeat doses, so does not pose significant problems for long term pain management.

\section{Cancer pain and choice of opioid}

"Cancer pain" can be complex. Causes include: direct tumour infiltration of pain sensitive structures, injuries resulting from cancer treatment (radiation, chemotherapy, or surgery) and vascular occlusion due to tumour or treatment effects. Physiologically, there are three types of pain:

(1) Somatic or nociceptive pain (arising from receptors in cutaneous or deep tissues such as bone).

\begin{tabular}{|c|c|c|}
\hline Opioid & Receptor action & Comments \\
\hline Morphine & Mu agonist & $\begin{array}{l}\text { Metabolised in liver to morphine-3 and morphine- } 6 \text { glucuronides (M3G and M6G). M6G } \\
\text { metabolite more potent than morphine }\end{array}$ \\
\hline Hydromorphone & Mu agonist & Morphine analogue \\
\hline Fentanyl & Mu agonist & \\
\hline Oxycodone & $\mathrm{Mu}$ agonist & \\
\hline Pentazocine & Kappa agonist; weak mu antagonist & $\begin{array}{l}\text { Multiple receptor activity: kappa effects analgesic but also increased psychotomimetic } \\
\text { effects cf mu agonists. Mu receptor antagonism can precipitate withdrawal if given } \\
\text { alongside mu agonist }\end{array}$ \\
\hline Methadone & $\begin{array}{l}\text { Mixed mu/delta agonist; } N \text {-methyl-D-aspartate } \\
\text { (NMDA) receptor antagonist }\end{array}$ & Multiple receptor activity—may act on complex pain \\
\hline Buprenorphine & Partial mu agonist; kappa antagonist; delta agonist & Complex receptor action—has dose ceiling for analgesic effect and antagonises other opioids \\
\hline Tramadol & Opioid plus serotonergic effects & For moderate pain. Tricyclic-like action - may act on neuropathic pain \\
\hline
\end{tabular}




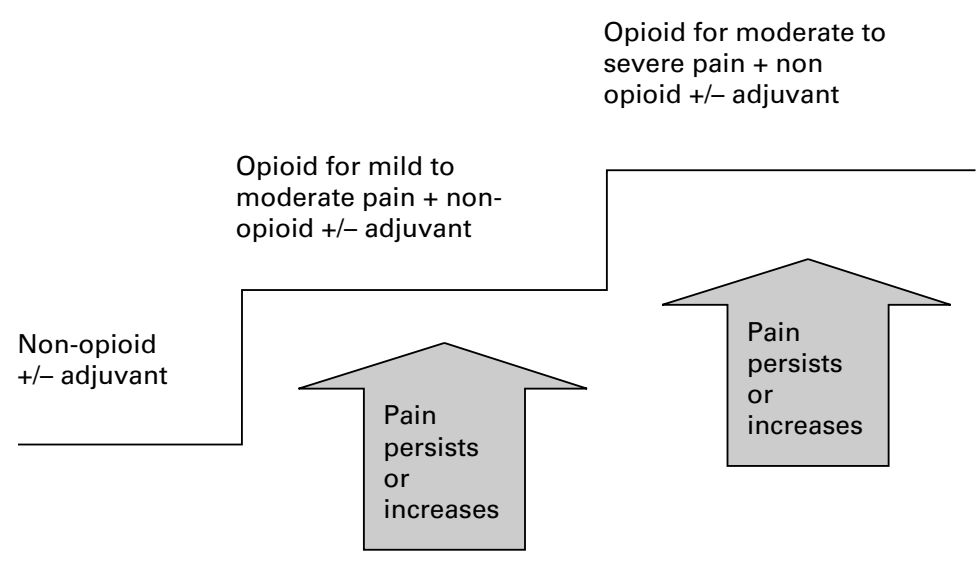

Figure 1 WHO analgesic ladder (adapted from $\mathrm{WHO}^{2}$ ).

(2) Visceral pain (arising from internal organ involvement).

(3) Neuropathic pain (arising from peripheral or central nervous systems).

Most pain can be controlled by pharmacological means, but it is essential to choose the right drugs for the individual. To help simplify approaches to pain control, the World Health Organisation (WHO) developed a three step analgesic ladder (fig 1). The fundamental principles are that:

(1) Inadequate pain control at one level requires a move to the next level, not to an alternative drug of the same efficacy.

(2) Continuous pain requires continuous analgesia.

The steps are simple:

(1) Treatment of mild pain is initiated with non-opioid analgesics (for example, paracetamol).

(2) Moderate pain that is not controlled by non-opioids should be treated by a weak opioid (alone or in combination with a non-opioid, for example, coproxamol: paracetamol and dextropopoxyphene).

(3) Severe pain should be treated by strong opioids.

At all levels adjuvant drugs can be added for specific indications: non-steroidal antiinflammatory drugs (NSAIDs) for bone pain; anticonvulsants or tricyclics for neuropathic pain. This is, however, a large subject in its own right, and will not be dealt with further here.

Table 3 A selection of opioids in common use in the UK (from the British National Formulary)

\begin{tabular}{ll}
\hline (1) Weak opioids & (2) Moderate and strong opioids \\
\hline Dextropopoxyphene & Buprenorphine (Temgesic) \\
Codeine & Dextromoramide (Palfium) \\
Dihydrocodeine & Diamorphine \\
& Dipipanone (Diconal) \\
& Fentanyl \\
& Hydromorphone (Palladone) \\
& Methadone \\
& Morphine \\
& Oxycodone \\
& Pentazocine (Fortral) \\
& Pethidine \\
& Phenazocine (Narphen) \\
& Tramadol \\
\hline
\end{tabular}

Table 4 Relative potency of commonly used opioids

\begin{tabular}{lll}
\hline Analgesic & $\begin{array}{l}\text { Potency ratio with } \\
\text { oral morphine }\end{array}$ & $\begin{array}{l}\text { Duration of } \\
\text { action (hours) }\end{array}$ \\
\hline Codeine & $1 / 10$ & $3-5$ \\
Pethidine & $1 / 8$ & $2-3$ \\
Tramadol & $1 / 5$ & $5-6$ \\
Dipipanone & $1 / 2$ & $3-5$ \\
Oxycodone & $1.5-2$ & $5-6$ \\
Dextromoramide & 2 & $2-3$ \\
Phenazocine & 5 & $6-8$ \\
Methadone & $5-10$ & $8-12$ \\
Hydromorphone & 7.5 & $3-5$ \\
Buprenorphine & 60 & $6-8$ \\
Fentanyl & 150 & 72 \\
\hline
\end{tabular}

\section{Choosing an appropriate opioid}

Several factors influence choice of an appropriate opioid.

- Availability

- Drug profile

- Individual patient factors

- Possible/desirable routes of administration

- Comparative analgesic effects

- Comparative adverse effects

- Other potential therapeutic effects

AVAILABILITY

In the UK, there is a wide range of opioids available (table 3 ). The initial choice of weak, moderate, or strong opioid is determined after careful assessment of the individual patient. It cannot be emphasised enough that pain is multifactorial and that successful treatment depends on comprehensive evaluation.

For the purposes of this review, I will focus discussion on strong opioids.

DRUG PROFILE

In palliation, the aim is to administer effective analgesics with a half life of several hours so that pain can be quickly controlled. Once dose requirements have stabilised, modified release formulations are extremely helpful, allowing longer dose intervals but maintaining flexibility to make dose alterations without risk of accumulation. Thus potency and duration of action are major determining factors. Morphine provides the gold standard: in unmodified form its four hour clinical duration of action allows regular review of pain control. Once stable, sustained release formulations reduce dose frequency to once or twice daily. Breakthrough pain is controlled with extra doses of the unmodified drug (calculated as $1 / 6$ of the total 24 hour opioid dose requirement). Drugs with a very short half life (for example, pethidine) are unsuitable, because of the need for more frequent repeat dosing, which is both inconvenient and can cause build-up of toxic metabolites. Drugs with inherently long half lives (for example, methadone), are useful for long term maintenance, but can be difficult to titrate safely in unstable pain. Table 4 illustrates the relative potency of various opioids compared with oral morphine, and also their duration of action.

\section{PATIENT FACTORS}

Biomedical

These include age related changes in metabolism and concurrent medical conditions. In palliative care, many patients are both elderly and have concurrent medical conditions, both of which 
Table 5 Important clinical drug interactions with opioids

\begin{tabular}{|c|c|}
\hline Class of drug & Clinical effects of interaction \\
\hline Alcohol & Enhanced sedative and hypotensive effects (all opioids) \\
\hline Antiarrhythmics & Delayed absorption of mexiletine (all opioids) \\
\hline Antibacterials & $\begin{array}{l}\text { Rifampicin accelerates metabolism of methadone (reduced effect of opioid); ciprofloxacin plasma concentration reduced by } \\
\text { opioids (reduced effect of antibiotic) }\end{array}$ \\
\hline Antidepressants & $\begin{array}{l}\text { Possible central nervous system excitation or depression if opioids given alongside monoamine oxidase inhibitors; increased risk of } \\
\text { convulsions with selective serotonin reuptake inhibitors and tricyclics if tramadol coadministered }\end{array}$ \\
\hline Antiepileptics & Effect of tramadol reduced by carbamazepine; phenytoin accelerates methadone metabolism \\
\hline Antipsychotics & Enhanced sedative and hypotensive effects (all opioids) \\
\hline Anxiolytics and hypnotics & Enhanced sedative effect (all opioids) \\
\hline Dopaminergics & Metaclopramide and domperidone antagonise gastrointestinal effects of opioids \\
\hline Ulcer healing drugs & Cimetidine inhibits metabolism of opioids (increased plasma concentration) \\
\hline
\end{tabular}

may influence the pharmacokinetics of opioids. For example cirrhosis affects bioavailability due to reduction of the first-pass effect. However, renal impairment is most important, as it affects clearance of many opioids. Reduced renal clearance is also the most important age related change, occurring in the elderly independently of specific disease conditions.

Therefore this group is particularly prone to accumulation of drugs at conventional dose intervals (the British National Formulary suggests by upwards of $\left.50 \%{ }^{3}\right)$. However, caution should not lead to inadequate analgesia, as all patients should have their pain requirements titrated individually. In practice, starting doses in uncontrolled pain usually remain the same, but patients with stable pain requirements and reduced renal clearance may require longer dose intervals or smaller doses.

Concurrent drug therapy can also alter opioid pharmacokinetics, for example, morphine and amitriptyline interact to produce increased bioavailability of the opioid, whereas coadministration of methadone and phenytoin leads to faster elimination of methadone (see table 5 for further examples). It is perhaps the uncommon interactions which should be remembered; others, while commonly warned against are often less relevant in clinical practice in palliative care, for example, alcohol in moderate doses rarely causes problems and enhances psychosocial well being.

Genetic factors predisposing to differences in opioid receptor response may be important, ${ }^{4}$ although these yet remain to be categorised.

\section{Psychosocial}

Patient acceptability-Acceptability is paramount to ensure compliance and ultimately effectiveness. At one end of the scale acceptability may be determined by simple factors such as taste or ease of swallowing; at the other end are complex issues such as the patient's previous analgesic experience or individual health beliefs. This needs to be explored sensitively to encourage doubts or concerns to be voiced.

Table 7 Comparative drug portraits

\begin{tabular}{llll}
\hline & Morphine & Hydromorphone & Methadone \\
\hline Origin & Main opium constituent & Semisynthetic opioid & Synthetic opioid \\
Oral absorption & 20 mins (10-30) & 30 mins & 3 hours (1-5 hours) \\
Oral bioavailability & About 40\% & About $50 \%$ & About $80 \%$ \\
Liver metabolites & M6G active & HM3G probably inactive & N-Demethylation: M1 and M2 both inactive \\
& M3G inactive & Kidney & Kidney (increased by acid urine) and faeces \\
Excretion & Kidney & 3-4 hours & Acute $8-20$ hours \\
Half life & Oral 2-2.5 hours & 4 hours & Chronic 16-29 hours \\
Useful analgesia & $3-6$ hours & & Variable \\
\hline
\end{tabular}

Many older patients in particular view the introduction of strong opioids as "the end of the road". They may be frightened of taking strong painkillers "too soon", believing they will no longer work when their illness progresses; this may lead them to downplay their symptoms. They may also have fears either of addiction or overdose. Recent high profile murder trials may have exacerbated confusion over the difference between deliberate fatal overdosage and the side effects of therapeutic opioid levels. ${ }^{5}$ While hopefully most people still trust their doctors' motives, there may yet be a knock-on effect. ${ }^{6}$

Many patients also have concerns about their ability to function normally when taking opioids. It is worthwhile negotiating and planning the introduction of an opioid to minimise any negative impact on the patient's life.

Patient safety - Other factors to consider are physical limitations (such as poor sight) or cognitive impairment, especially among patients living alone, where safety as well as compliance may be an issue.

\section{ROUTES OF ADMINISTRATION}

Opioids can be given by a variety of routes (table 6). While most opioids can be given orally, a different route requirement may affect choice of drug. It should be noted that the intramuscular route has not been included. In the palliative care setting, this is rarely necessary or appropriate: it can be painful, particularly for debilitated patients with wasted muscles. In comparison, the subcutaneous route is more comfortable yet equally effective.

\section{Oral}

The most commonly used route is oral. As most opioids are available in oral form, choice is determined by the comparative efficacy, bioavailability, and side effect profile of different drugs. A comparison of morphine with two other commonly available strong opioids is given in table 7 .

\begin{tabular}{l} 
Table 6 Routes of admin- \\
istration of opioids in the \\
palliative care setting \\
\hline Oral \\
Sublingual \\
Subcutaneous \\
Intravenous \\
Epidural/intrathecal \\
Transdermal \\
Rectal
\end{tabular}


Hydromorphone-Hydromorphone is relatively new on the market, aimed as an alternative to oral morphine. ${ }^{7}$ It has a similar pharmacology to morphine but has inactive metabolites, which may explain its being better tolerated in selected patients. It is formulated in four hourly unmodified and 12 hourly modified release tablets, simplifying the transition from morphine, although the conversion ratio can be off-putting (7.5:1, that is, $7.5 \mathrm{mg}$ morphine is equipotent to $1 \mathrm{mg}$ of hydromorphone). For opioid responsive pain, it is comparable with morphine, and anecdotally, has less severe side effects; however, its similar receptor affinity does not allow this to be predicted for individual patients, and clinical trials to date have failed to demonstrate a clear superiority over morphine. It therefore tends to be used as a second-line alternative, or in "opioid rotation".

"Opioid rotation" means switching from one strong opioid to another where pain management is requiring increasing dose escalation, as patients may achieve better analgesia and/or reduced toxicity with an alternative drug. ${ }^{8}$

A baseline figure for this is where the dose of oral morphine has reached $>1 \mathrm{~g} / 24$ hours. In some centres this is taken a step further with patients switched between opioids at regular intervals to minimise the development of analgesic tolerance. ${ }^{9}$ Opioid rotation is an area of increasing research interest in palliative care. ${ }^{10}$

Methadone-Methadone is an interesting drug that has recently come back into vogue in palliative care. ${ }^{11}$ Previously it was considered pharmacologically "dirty": its broad based receptor activity, combined with a complex biodistribution led to difficulties in dose titration. While these considerations remain, it may have a particular place in treating complex pains. Indications include nociceptive pain and opioid responsive pain with neuropathic elements, such as intractable facial pain.

It is slowly eliminated, reducing the incidence of breakthrough pain, yet does not accumulate in renal impairment, and shows no cross tolerance with other opioids. Thus it can be used in cases of true morphine allergy (although relatively uncommon) and is useful for opioid rotation. ${ }^{12}$

Its disadvantages represent the flip side to its advantages. While the half life of the free drug is measured in hours, only a small fraction is present in the circulation, the greater part passing into a much larger tissue reservoir. Reliable dosing is only achieved when these fractions have reached equilibrium. This makes initial dose titration difficult, particularly the risk of overdose. There are also conversion difficulties (if opioid rotating) because of the absence of cross tolerance. Breakthrough pain can also be difficult to treat because of the drug's slow onset of action. Specialist supervision is therefore advisable for the first two weeks or so, usually as an inpatient, although it has been reported in the outpatient setting, with careful monitoring. ${ }^{13}$

The other disadvantage is that if patients cannot take the oral form, subcutaneous administration is generally not advised because of irritant effects, although recent work has suggested options to alleviate irritation and allow continued use of methadone. ${ }^{14}$

Perhaps because it has been neglected in the past and because of its variable half life, there has been a lack of consensus on appropriate protocols, although this has improved with wider usage. ${ }^{15}$ Methadone is perhaps best viewed as a useful second line drug in the specialist palliative care setting.

Tramadol and oxycodone are two other drugs that have recently been strongly marketed. Tramadol is a relatively recent arrival, which inhibits serotonin reuptake in addition to its weak mu receptor agonist action; this makes it potentially useful for opioid responsive pain with neuropathic elements. This has been demonstrated in the management of polyneuropathy. ${ }^{16}$ It also has less effect on gastric stasis. However, although it provides a useful alternative at high dose in moderate pain, ${ }^{17}$ it is less potent than morphine and less effective for managing severe pain. ${ }^{18}$ Its current place in palliative care is therefore unclear.

Oxycodone is a long established drug recently relaunched in new sustained release oral formulations. It is reputed to be less likely to cause hallucinations and delirium. ${ }^{19}$ It has been used successfully in patients with advanced cancer, ${ }^{20}$ but may not be totally equivalent in analgesic efficacy to morphine, ${ }^{21}$ although comparison of controlled release preparations in stable pain showed no difference. $^{22}$

\section{Sublingual}

This could be useful in theory, particularly for patients with swallowing difficulties. However, only buprenorphine is so formulated. As a partial analgesic agonist which antagonises other opioids, it is difficult to use in the palliative care setting and is not recommended.

\section{Rectal}

This route was much favoured in the past, particularly in nursing homes, because it provided a reliable route for non-specialist nurses treating semiconscious patients who were unable to swallow. With the development of syringe drivers and transdermal formulations it is now less commonly used.

Although a variant on the gastrointestinal route, rectal administration may affect bioavailability due to partial bypassing of hepatic metabolism. In the opioid naïve patient, there is some evidence of both more rapid and sustained pain control when comparing rectal with oral administration of morphine. ${ }^{23}$ There is however little published on long term use for cancer pain. ${ }^{24}$

Prepared rectal formulations (that is, suppositories) include morphine, hydromorphone, and oxycodone. Opioids have also been administered rectally in non-standard preparations, usually liquid for fast absorption.

\section{Subcutaneous}

This route has increased in popularity over the last 15 years, as clinicians have come to appreciate its flexibility, safety, and practicality. It is 
now the first choice in most instances where the parenteral route is required.

In the community setting it has revolutionised patient care, both in the terminal stages and for those with dysphagia, vomiting, impaired absorption, or obstruction. The crucial development has been the syringe driver. ${ }^{25}$ This simple device utilises normal plastic syringes, the rate of flow being preset (usually to run over 24 hours). Being battery driven, it is relatively unobtrusive and does not affect mobility. It offers a reliable constant route of administration; at the same time subcutaneous absorption is partially rate limited (compared with intravenous route), so reducing the risk of inadvertent overdose, making it safer for use in unsupervised settings. Setting up and recharging the driver are straightforward procedures for trained district nurses.

The opioids most commonly employed are diamorphine (UK), morphine (US), or more recently hydromorphone. The avoidance of first-pass metabolism increases bioavailability, although there is some variation in practice as to actual dosage regimens. The majority of centres in the UK use a 3:1 ratio (that is, 300 mg oral morphine over 24 hours converts to $100 \mathrm{mg}$ subcutaneous diamorphine).

Problems with subcutaneous administration usually relate to skin sites, most commonly when using high concentrations of opioid, or when combining opioid with other drugs (compatibility guidelines are available). Irritation can often be overcome by reducing the concentration of drugs (using a larger volume) or by adding hydrocortisone to the infusion. Similarly problems with absorption can be ameliorated by adding hyaluronidase (an enzyme that breaks down connective tissue and increases local diffusion). Other occasional problems include localised needle reactions (an alternative Teflon cannula is available), while in severely cachectic patients, siting may be difficult due to lack of subcutaneous tissue. Very occasionally, shutdown of the patient's peripheral circulation in the terminal phase may cause unreliable absorption, requiring more frequent review and appropriate dose adjustments to prevent distress.

\section{Intravenous}

This route is not commonly used now: bolus administration provides rapid onset (10-15 mins for morphine) but short duration of action of analgesia and is therefore only used for emergency symptom control. Continuous intravenous infusion is useful for control of severe pain where the subcutaneous route is not tolerated, particularly for dose titration over a relatively short period. Most intravenous pumps are unwieldy and intrusive, requiring mains attachment, so constraining mobility and longer term acceptability.

Patient controlled analgesia-This has been widely used intravenously in the acute setting for control of postoperative pain, pain associated with bone marrow transplants, and in
Summary of factors influencing choice of opioid

- Opioid sensitivity of pain - although obvious, there is a fundamental requirement to assess the nature of the presenting pain before prescribing an opioid at all.

- Stability of pain control-relevant in determining the formulation to be used.

- Presence of coexisting symptoms.

- Presence of concurrent factors affecting metabolism and drug elimination.

- Necessary or preferred route of administration.

- Patient psychosocial factors.

gastrointestinal obstruction where severe spasmodic pain may exacerbate lower levels of background pain.

More recently the Edmonton Injector ${ }^{26}$ has been developed to allow subcutaneous bolus injection, leading to more widescale use in the community setting in North America, although this has not yet found favour in the UK.

\section{Epiduralintrathecal}

Spinal routes of administration are used commonly for inpatients. Community use is possible but more problematic because of lack of trained back-up.

The main applications of this route are twofold:

(1) Providing longer duration of analgesia at a lower overall dose, because of greatly increased bioavailability, and hence reduced unwanted peripheral side effects.

(2) Useful for controlling severe localised lower body pain, for example, pelvic disease.

Disadvantages are:

(1) Risk of infection, minimised by tunnelling the catheter.

(2) Risk of overdose if breakthrough doses are given by inexperienced staff; this can be avoided as long as pain control is stable before discharge, as oral administration can still be used for occasional breakthrough requirements.

In Britain diamorphine remains the drug of choice, because of its high solubility, with morphine used elsewhere. More controlled trials are required to establish the place of other opioids.

\section{Transdermal}

The transdermal route (that is, skin patch) is another relatively recent development in pain control: only fentanyl is currently available in this formulation.

Fentanyl is a highly potent mu receptor opioid with good comparative clinical analgesic efficacy to morphine. ${ }^{27}$ Main indications for its use are where oral administration is not possible for reasons such as dysphagia, vomiting, or impaired gastrointestinal absorption, that is, similar to indications for the subcutaneous route. Fentanyl is reported to have less gastrointestinal side effects than other opioids. It may therefore be helpful when nausea and vomiting is drug related, or in severe constipation. ${ }^{28}$ 
It should only be used after titration with short acting opioids to stabilise pain, as the slow equilibration of blood levels transdermally makes it unsuitable for short term review.

The advantages of transdermal administration are: it is generally highly acceptable to patients; patches can be applied by patients or relatives themselves; the long duration of action requires infrequent patch changes, minimising non-compliance in the community and reducing the time spent by ward staff administering controlled drugs.

Fentanyl may also be more acceptable both to patients and physicians because of its lack of association with morphine, enhanced by perception of the transdermal route as less "medical". This may encourage individual patients to accept more effective pain control, but should not be a substitute for education among clinicians anxious about prescribing appropriate doses of opioids.

The downside of viewing fentanyl as "innocuous" is that patients may be prescribed a patch de novo after weak opioids only, without assessment of opioid requirement. This can cause problems of overdosage and side effects, particularly because the morphine equivalent dose effect of the patches demonstrates considerable and unpredictable interpatient variability - the $25 \mu \mathrm{g} /$ hour patch being equivalent to between 30 and $135 \mathrm{mg}$ of oral morphine over 24 hours! The other problem with using patches de novo in unstable pain is the slow titration to analgesic requirements, with patients still requiring breakthrough doses of another strong opioid, as fentanyl is not currently available for this.

Other disadvantages are its long half life prolonging the duration of action after patch removal, and so effects in overdose, and complicating opioid rotation. Its duration of action also makes it less flexible for circadian ${ }^{29}$ or other individual variations in analgesic requirement. Finally, it is relatively expensive. While cost considerations should never prevent appropriate use, overenthusiastic prescribing for inappropriate reasons (such as avoidance of a discussion about morphine) is not to be condoned.

\section{Limitations of existing evidence}

While the above can be a guide, it may be more difficult to make systematic choices in practice, because reviews of published evidence reveal several limitations:

- Most studies have focused on morphine itself.

- Most studies of other opioids focus on "new" drugs or new product licences.

- Single drug studies have considered only analgesic efficacy and acceptability.

- Comparative studies have focused on establishing efficacy/potency of analgesic action.

So what's missing? There are few large scale comparative trials, and most have focused on analgesia. However, to use different opioids in a more sophisticated way it is the other opioid effects which may swing the balance, and it is in this area that least work has been done:

\section{Questions (see p 377 for answers)}

(1) What are the principal opioid receptor sites, and which physiological effects are mediated by each?

(2) How does methadone differ from other opioids in its receptor activation, and what is the clinical significance of this?

(3) Why is methadone difficult to titrate?

(4) Name two routes of administration other than oral which are important in palliative care.

(5) What potential advantages does fentanyl appear to demonstrate in terms of side effects?

(6) Are opioids useful for the treatment of dyspnoea?

- Studies comparing effects of different routes of administration of the same opioid are lacking.

- Systematic comparison and quantification of adverse effects between opioids is lacking, for example, there are reports of reduced adverse gastrointestinal side effects with fentanyl and hydromorphone in comparison with oral morphine, but this needs more systematic exploration.

- Studies comparing analgesic effects of opioids in treating complex pain. This is particularly relevant to methadone, whose $N$-methyl-D-aspartate (NMDA) receptor antagonist action may make it more effective for neuropathic-type pain. However, no clinical studies have been carried out to examine this systematically. ${ }^{30}$

Finally, an area which is almost completely unexplored:

- Studies comparing potentially beneficial side effects between opioids, for example, impact on dyspnoea.

In this example, initial experience using inhaled morphine has suggested that dyspnoea could be improved independent of any analgesic requirement, ${ }^{31} 32$ while a small randomised controlled trial failed to reach significance, but did report strong treatment effects in individual patients. ${ }^{33}$ Studies of inhaled morphine have demonstrated rapid systemic absorption yet a lack of effect on dyspnoea, ${ }^{34}$ while a small study of patients receiving oral morphine demonstrated symptomatic improvement in breathlessness alongside systemic side effects. ${ }^{35}$ Thus it is not clear whether the response is due to morphine acting at local receptor level in the lungs or through central inhibition combined with reduction of anxiety. If that is the picture regarding morphine, it is not surprising that to date no comparison studies have emerged. Clearly, more work needs to be done on dyspnoea and opioids.

\section{Questions we might like to ask}

At present, we cannot effectively predict the response to an individual opioid for an 


\section{Answers}

(1) $\mathrm{Mu}$, kappa and delta - analgesia is mediated by all three, while nausea, sedation, and constipation are primarily through mu receptor activity, and dysphoria and psychotomimetic effects through the kappa receptor.

(2) Methadone has an additional action as an NMDA receptor antagonist - the clinical significance of this is that it may confer improved analgesic effects when treating complex pains with a neuropathic element.

(3) Methadone is difficult to titrate because it has a complex biodistribution, with a small free plasma fraction and large tissue reservoir.

(4) Subcutaneous and transdermal routes.

(5) Fentanyl appears to have less constipating effects than other opioids.

(6) The jury's still out on this one-small scale trials have suggested clinical benefits exist, but larger randomised trials have yet to be conducted.

individual patient. These gaps in our knowledge lead to a number of specific questions for further research:

- What are the comparative effects of different opioids in alleviating breathlessness? For example, morphine $v$ methadone $v$ fentanyl.

- What is the most logical choice of short acting opioid to use for breakthrough pain when administering transdermal fentanyl?

- Are there really significant differences between opioids with respect to causing constipation?

- Does methadone have a specific place in cancer pain management?

At present none of these questions can be answered. Multicentre trials in palliative care have been slow to develop. There are practical issues of funding and coordinating trials among a large number of small units, and difficulties in designing scientifically robust protocols that fulfil the needs of terminally ill patients both clinically and ethically. Recruitment tends to be slow, attrition rates high, and assessment of effects confounded by patients having multiple problems and requiring different treatment modalities. However, comparative work undoubtedly needs to be done if we are aiming to offer patients informed choice and optimal symptom control. In end stage disease there is no time to waste in trial and error. Among earlier stage patients or those with non-malignant chronic pain, improved understanding will improve care and maximise patients' functional capacity and quality of life.

The author thanks Benoit Ritzenthaler, Consultant in Palliative Medicine, Compton Hospice, Wolverhampton for a personal communication on methadone; Carol Davies, Senior Lecturer in Palliative Medicine, Countess Mountbatten House, Southampton for discussion of review undertaken by Carla Ripamonti ampton for discussion of review undertaken by Carla Ripamonti
on behalf of EAPC Working Group on Opioid Rotation and for summary of their initial conclusions (October 1998); and the Napp Information Service for additional searches and supply of monographs on hydromorphone.

Literature search: Netscape search of Medline using search terms "palliative care and opiates"; "opiates and cancer"; "opiates and dyspnoea".

Further reading: Oxford Textbook of Palliative Medicine. Doyle D, Hanks GWC, MacDonald N, eds. 1st Ed (reprint in paperback; 3rd edition in press), Oxford University Press, 1996; Symptom management in advanced cancer. Twycross R. 2nd Ed, C, Carter Y, Woof R, eds, Blackwell Science, 1998.

1 Bruera E, Fainsinger R, MacEachern T, et al. The use of methylphenidate in patients with incident cancer pain
receiving regular opiates. A preliminary report. Pain 1992;50:75-7.

2 Report of a WHO Expert Committee. Cancer pain relief and palliative care. Geneva: World Health Organisation, 1990 (WHO Technical Report Series, No 804)

3 British Medical Association and Royal Pharmaceutical Society of Great Britain. Prescribing for the elderly. British National Formulary. London: BMA, March 2000;39:16-17.

4 Galer BS, Coyle N, Pasternak GW, et al. Individual variability in the response to different opioids: report of five cases. Pain 1992;49:87-91.

5 O'Neill B. Doctor as murderer [editorial]. BMF 2000;320: 329-30.

6 Pringle M. The Shipman inquiry: implications for the public's trust in doctors [editorial]. Br f Gen Pract 2000;50:355-

7 McDonald CJ, Kelly DG, Miller AJ. Hydromorphone. CME McDonald CJ, Kelly DG, Miller AJ.Hydr
Bulletin Palliative Medicine 1998:1(1).

8 de Stoutz ND, Bruera E, Suarez-Almazor M. Opioid de Stoutz ND, Bruera E, Suarez-Almazor $\mathrm{M}$. Opioid Pain Symptom Manage 1995;10:378-84.

9 Fainsinger RL, Louie K, Belzile M, et al. Decreased opioid doses used on a palliative care unit. $\mathcal{F}$ Palliat Care 1996;12:6-9.

10 Mercadante S. Opioid rotation for cancer pain: rationale and clinical aspects. Cancer 1999;86:1856-66.

11 Gannon C. The use of methadone in the care of the dying. European fournal of Palliative Care 1997;4:152-7.

12 Crews JC, Sweeney NJ, Denson DD. Clinical efficacy of methadone in patients refractory to other mu-opioid receptor agonist analgesics for management of terminal cancer pain. Case presentations and discussion of incomplete cross-tolerance among opioid agonist analgesics. Cancer 1993;72:2266-72.

13 Hagen NA, Wasylenko E. Methadone: outpatient titration and monitoring strategies in cancer patients. $f$ Pain and monitoring strategies in can

14 Mathew P, Storey P. Subcutaneous methadone in terminally ill patients: manageable local toxicity. F Pain Symptom Manage 1999;18:49-52

15 Morley JS, Makin MK. The use of methadone in cancer pain poorly responsive to other opioids. Pain Reviews 1998; 5:51-8.

16 Sindrup SH, Andersen G, Madsen C, et al. Tramadol relieves pain and allodynia in polyneuropathy: a randomised, double-blind, controlled trial. Pain 1999;83:8590 .

17 Grond S, Radbruch L, Meuser T, et al. High-dose tramadol in comparison to low-dose morphine for cancer pain relief. f Pain Symptom Manage 1999;18:174-9.

18 Wilder-Smith CH, Schimke J, Osterwalder B, et al. Oral traWilder-Smith $\mathrm{CH}$, Schimke J, Osterwalder B, et al. Oral tra-
madol, a mu-opioid agonist and monamine reuptakemadol, a mu-opioid agonist and monamine reuptakeblocker, and morphine

19 Maddocks I, Somogyi A, Abbott F, et al. Attenuation of morphine-induced delirium in palliative care by substitution with infusion of oxycodone. F Pain Symptom Manage 1996; 12:182-9.

20 Glare PA, Walsh TD. Dose-ranging study of oxycodone for chronic pain in advanced cancer. F Clin Oncol 1993;11:9738.

21 Heiskanen T, Kalso E. Controlled-release oxycodone and morphine in cancer related pain. Pain 1997;73:37-45.

22 Bruera E, Belzile M, Pituskin E, et al. Randomized doubleblind, cross-over trial comparing safety and efficacy of oral controlled-release oxycodone with controlled-release morphine in patients with cancer pain. F Clin Oncol 1998;16: phine in

23 De Conno F, Ripamonti C, Saita L, et al. Role of rectal route in treating cancer pain: a randomized crossover clinical trial of oral versus rectal morphine administration in opioidof oral versus rectal morphine administration in opioid8 .

24 Ripamonti C, Bruera E. Rectal, buccal and sublingual narcotics for the management of cancer pain. $\mathcal{F}$ Palliat Care 1991;7:30-5.

25 Oliver DJ. Syringe drivers in palliative care: a review. Palliat Med 1988;2:21-6.

26 Bruera E, MacMillan K, Hanson J, et al. The Edmonton injector: a simple device for patient-controlled subcutaneous analgesia. Pain 1991;44:167-9.

27 Ahmedzai S, Brooks D. Transdermal fentanyl versus sustained-release oral morphine in cancer pain: preference, efficacy and quality of life. I Pain Symptom Manage 1997;13:254-61.

28 Radbruch L, Sabatowski R, Loick G, et al. Constipation and Radbruch L, Sabatowski R, Loick G, et al. Constipation and
the use of laxatives: a comparison between transdermal fentanyl and oral morphine. Palliat Med 2000;14:111-9. 
29 Bruera E, Macmillan K, Kuehn N, et al. Circadian distribution of extra doses of narcotic analgesics in patients with

30 Ebert B, Thorkildsen C, Andersen S, et al. Opioid analgesics as noncompetitive N-methyl-D-aspartate (NMDA) antagonists. Biochem Pharmacol 1998;56:553-9.

31 Farncombe M, Chater S, Gillin A. The use of nebulized opioids for breathlessness: a chart review. Palliat Med 1994; 8:306-12.

32 Zeppetella G. Nebulized morphine in the palliation of dypsnoea. Palliat Med 1997;11:267-75.
33 Davis CL, Penn K, A'Hern R, et al. Single dose randomized controlled trial of nebulised morphine in patients with cancer related breathlessness. Palliat Med 1996;10:

34 Jankelson D, Hosseini K, Mather LE, et al. Lack of effect of high doses of inhaled morphine on exercise tolerance in chronic obstructive pulmonary disease. Eur Respir $f$ 1997;10:2270-4.

35 Boyd KJ, Kelly M. Oral morphine as symptomatic treatment of dyspnoea in patients with advanced cancer. Palliat Med 1997;11:277-81.

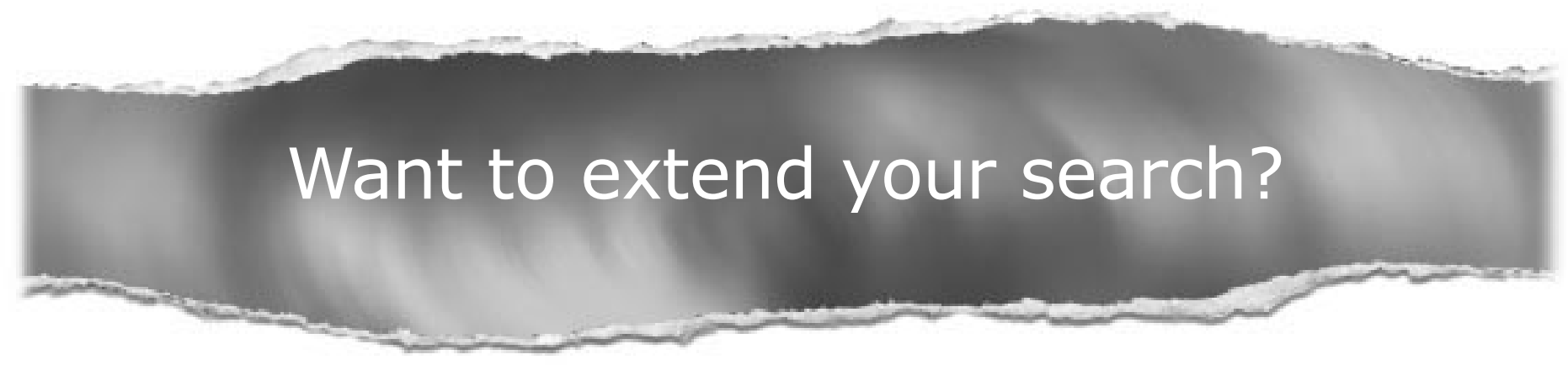

Cross journal searching

If you can't find what you are looking for in the Postgraduate Medical Journal you can extend your search across many of the more than $\mathbf{2 0 0}$ journals available for selection. You can restrict your search to specific subject areas (eg, clinical medicine, basic research), or select specific journals, or search all available titles.

www.postgradmedj.com 